\title{
Emergence and maintenance of biodiversity in an evolutionary food-web model
}

Åke Brännström (ake.brannstrom@math.umu.se)

Nicolas Loeuille (nicolas.loeuille@normalesup.org)

Michel Loreau (michel.loreau@mcgill.ca)

Ulf Dieckmann (dieckmann@iiasa.ac.at)

\section{Approved by}

Detlof Von Winterfeldt

Director

July 2011 


\title{
Emergence and maintenance of biodiversity in an evolutionary food-web model
}

\author{
Å. Brännström, N. Loeuille, M. Loreau, and U. Dieckmann
}

June 2010

\begin{abstract}
Ecological communities emerge as a consequence of gradual evolution, speciation, and immigration. In this study, we explore how these processes and the structure of the evolved food webs are affected by species-level properties. Using a model of biodiversity formation that is based on body size as the evolving trait and incorporates gradual evolution and adaptive radiation, we investigate how conditions for initial diversification relate to the eventual diversity of a food web. We also study how trophic interactions, interference competition, and energy availability affect a food web's maximum trophic level and contrast this with conditions for high diversity. We find that there is not always a positive relationship between conditions that promote initial diversification and eventual diversity, and that the most diverse food webs often do not have the highest trophic levels.
\end{abstract}

\section{Introduction}

There is mounting awareness of the essential role of species richness in ecosystem functioning (Naeem et al., 1994; Tilman et al, 1996; Hector et al., 1999; Díaz and Cabido, 2001; Loreau et al., 2001; Kinzig et al., 2001; Loreau et al., 2002; Hooper et al., 2005; Naeem et al., 2009). To understand the emergence and maintenance of species-rich communities, we need to consider the ways species are connected through ecological interactions. The networks formed by trophic interactions among a community's species are known as food webs. As a means of understanding levels of ecological organization, the study of food webs holds great promise and may provide insights important for tackling a number of hard ecological problems, including understanding potentially detrimental anthropogenic impacts on the diversity of life. By including additional processes, such as competitive interactions among species, the accuracy of the representation of an ecological community can be increased. However, despite decades of intense efforts, even basic questions such as those concerning the relationship between stability and complexity of communities are still vigorously contested (May, 1972; Pimm and Lawton, 1977; Ives et al., 2000; Jansen and Kokkoris, 2003; Kondoh, 2003; Worm and Duffy, 2003).

The structure of food webs arises from properties of individual species. These properties affect both the addition of new species through immigration from other ecosystems, and the gradual evolution and adaptive radiation of the 
species that are already present. The latter process may give rise to new species through evolutionary branching, but even without speciation the coevolution of species may change the structure of food webs by altering trophic interaction strengths (Saloniemi, 1993; Dieckmann and Law, 1996; Abrams, 1997; Bergelson et al., 2001; Buckling and Rainey, 2002a; Loeuille et al., 2002). While there is widespread consensus on the importance of linking species-level properties to the structure of food webs, theoretical understanding of food-web patterns is largely based on studies of community-level properties. For example, foodweb models such as the cascade model (Cohen et al., 1990), the niche model (Williams and Martinez, 2000), or the nested-hierarchy model (Cattin et al., 2004) often use fixed relationships among community-level properties of food webs, such as connectance and diversity. Although these approaches are useful, they cannot account for the effects of species abundances or interaction strengths on community-level properties, nor can they describe the dynamical properties of food webs.

For these reasons, several food-web models have been constructed that incorporate population dynamics. The first of these were species-assembly models (Post and Pimm, 1983; Drake, 1990; Law and Morton, 1996; Morton and Law, 1997), which assume the infrequent immigration of new species from a predetermined species pool. Such models can adequately describe how smaller food webs, for example on islands, originate through the immigration of species from a mainland, but do not in general provide an adequate description of the origin of food webs. More recently, food-web models have thus been constructed that that incorporate evolutionary processes. The common aim of these models is to reconcile species-level properties with emergent community-level properties of food-web structure and dynamics. The webworld model (Caldarelli et al. 1998; Drossel et al. 2001; McKane 2004) describes the evolutionary assembly of food webs based on a predetermined large number of binary traits. Tokita and Yasutomi (2006) generated rich food webs based on replicator dynamics, by allowing interaction strengths, rather than the underlying ecological traits, to evolve directly. Loeuille and Loreau (2005) constructed a model with a firm ecological grounding based on a single continuous trait, body size, and showed that complex food-web structures may emerge from simple ecological and evolutionary rules. The model by Loeuille and Loreau (2005) was the first dynamic model of food-web evolution that incorporated physiologically important aspects such as body size and metabolic scaling. Ito and Ikegami (2006) showed that two abstract continuous traits - characterizing, respectively, an individual's key quantitative features in its role as consumer and as resource - suffice for the evolutionary emergence of rich and diverse food webs. Rossberg et al. (2008) combined allometric scaling relationships with abstract vulnerability and foraging traits, resulting in models in which repeated invasions and large mutational steps lead to ecologically realistic food webs.

The principal aim of this study is to explore the relationship between factors determining species-level interactions and the properties of evolved communities. We present a model of biodiversity evolution that descends from Loeuille and Loreau (2005), but in which several of the underlying assumptions have been revisited and revised. The model incorporates gradual evolution (Dieckmann and Law, 1996), evolutionary branching (Geritz et al., 1998), and trophic interactions that depend on relative, rather than absolute, differences in body size. Also, as the revised model is based on small mutational steps, we can ex- 


\begin{tabular}{lll}
\hline Parameter & Description & Unit \\
\hline$d_{0}$ & Proportionality constant for morph death rate & $1 /$ time \\
$\lambda$ & Conversion efficiency & \\
$M_{\gamma}$ & Amplitude of consumption kernel & $1 /$ time \\
$\mu$ & Optimal relative body size for foraging & \\
$\sigma_{\gamma}$ & Standard deviation of consumption kernel & \\
$M_{\alpha}$ & Amplitude of competition kernel & $1 /$ time \\
$\sigma_{K}$ & Standard deviation of competition kernel & \\
$r_{g}$ & Growth rate of resource & $1 /$ time \\
$k_{0}$ & Strength of density dependence in the resource & $1 /$ time \\
$r_{i}$ & Trait value of morph $i>0$ & \\
$\sigma_{m}$ & Standard deviation of mutational steps & \\
$\tau$ & Mutation probability per birth event & \\
\hline
\end{tabular}

Table 1: Major parameters used in the ecological and evolutionary model. The first nine parameters affect the ecological dynamics while the last three parameters affect the evolutionary process

plore questions that cannot be addressed using models based on immigration or large mutational steps. Using this model, we explore how conditions for initial diversification relate to the eventual diversity of an evolving food web. We feel that this question is very timely. Many recent works have studied conditions for the emergence of diversity (e.g. Rainey and Travisano, 1998; Buckling and Rainey, 2002b; Nosil and Crespi, 2006), but how these relate to conditions for the maintenance of diversity in natural communities remains largely unknown. Finally, we investigate how trophic interactions, interference competition, and energy availability affect a food web's maximum trophic level and contrast this with conditions for high diversity.

\section{Model description}

We consider one basal autotrophic resource $(i=0)$ and $n$ heterotrophic morphs $(i=1, \ldots, n)$ with positive densities $x_{i}$. Here and below, we use the term morph, instead of species, to reflect that our model is not concerned with reproductive isolation, or its evolution. To each morph $i$ we associate a value $s_{i}$ that we interpret as the average mass of an individual of that morph. Since many ecological relationships involving body sizes are more conveniently expressed in terms of relative rather than absolute differences, we work on a logarithmic scale and define $r_{i}=\ln \left(s_{i} / s_{0}\right)$ as the trait value of an individual of morph $i$, where $s_{i}$ is normalized by the size $s_{0}$ of the basal autotrophic resource. 


\section{Demographic dynamics}

The dynamics of the $n$ heterotrophic morphs are governed by the Lotka-Volterra equations

$$
\begin{aligned}
\frac{\dot{x_{i}}}{x_{i}}= & -d\left(r_{i}\right)+\sum_{j=0}^{n} \exp \left(-r_{i}\right) \lambda \exp \left(r_{j}\right) M_{\gamma} \gamma\left(r_{i}-r_{j}\right) x_{j} \\
& -\sum_{j=1}^{n} M_{\gamma} \gamma\left(r_{j}-r_{i}\right) x_{j}-\sum_{j=1}^{n} M_{\alpha} \alpha\left(r_{i}-r_{j}\right) x_{j} .
\end{aligned}
$$

Here, $\dot{x}_{i}$ denotes the derivative of $x_{i}$ with respect to time and the terms on the right-hand side are per capita rates corresponding, from left to right, to intrinsic mortality, reproduction, mortality from predation, and mortality from interference competition. The first of these, the intrinsic death rate, is assumed to decline exponentially with $r_{i}, d\left(r_{i}\right)=d_{0} \exp \left(-q r_{i}\right)$. Although there is not much empirical data linking body mass with intrinsic mortality, Peters (1983) suggests an exponent of $q=1 / 4$ based on the allometric relationship between generation time and body mass. Alternatively, if we express the equations above in terms of biomasses rather than densities, the first term may be seen as describing the loss of biomass through respiration, corresponding to an allometric scaling of metabolic rate with body mass with an exponent of $3 / 4$.

The reproductive growth rate, or birth rate, $B_{i}$ is a product of several factors. First, $M_{\gamma} \gamma\left(r_{i}-r_{j}\right) x_{j}$ is the rate at which individuals of morph $j$ are ingested by those of morph $i$. Multiplying this with the body mass $s_{0} \exp \left(r_{j}\right)$ of the consumed individuals gives the consumer's intake rate of biomass, of which a fraction $\lambda$ can be used for the consumer's reproductive growth. The reproductive growth rate is then obtained by dividing this by the body mass $s_{0} \exp \left(r_{i}\right)$ of the consumer, canceling out the common factor $s_{0}$. We take $\gamma$ to be the probability density function of the normal distribution. Writing

$$
M_{\gamma} \gamma\left(r_{i}-r_{j}\right) x_{j}=\frac{M_{\gamma} x_{j}}{\sqrt{2 \pi} \sigma_{\gamma}} \exp \left(-\frac{\left(r_{i}-r_{j}-\mu\right)^{2}}{2 \sigma_{\gamma}^{2}}\right),
$$

the first factor on the right-hand side can be interpreted as the consumer's per capita encounter rate of individuals of morph $j$, which is proportional to the density $x_{j}$ with a constant of proportionality depending on $\sigma_{\gamma}$. The last factor can be interpreted as the probability that an individual of morph $j$ will be successfully attacked by a consumer of morph $i$. With this interpretation, decreasing $\sigma_{\gamma}$ will make attacks on individuals with trait values close to $\mu$ more successful, while at the same time increasing the encounter rate of all prey morphs. The conversion efficiency $0<\lambda<1$ measures the fraction of biomass of morph $j$ that morph $i$ can use for its reproductive growth.

The mortality rate from interference competition with other morphs is assumed to depend linearly on the population densities of the two interfering morphs, with the constant of proportionality given by a competition kernel $M_{\alpha} \alpha\left(r_{i}-r_{j}\right)$ defined by

$$
M_{\alpha} \alpha\left(r_{i}-r_{j}\right)=\frac{M_{\alpha}}{\sqrt{2 \pi} \sigma_{K}} \exp \left(-\frac{\left(r_{i}-r_{j}\right)^{2}}{2 \sigma_{K}^{2}}\right) .
$$


The intensity of interference competition is thus highest between individuals with the same body size and declines with relative difference in body size.

The resource dynamics are governed by the equation

$$
\frac{\dot{x_{0}}}{x_{0}}=r_{g}-\sum_{j=1}^{n} M_{\gamma} \gamma\left(r_{j}\right) x_{j}-k_{0} x_{0} .
$$

Excluding trait values, the ecological model comprises nine parameters, of which four are dimensionless. All parameters are listed in Table 1. Since the model involves two independent units, the number of parameters can be reduced to $9-2=7$, yielding the non-dimensionalized equations

$$
\frac{\dot{y}_{0}}{y_{0}}=1-a \sum_{j=1}^{n} \gamma\left(r_{j}\right) y_{j}-b y_{0},
$$

and

$$
\begin{gathered}
\frac{\dot{y}_{i}}{y_{i}}=-b \exp \left(-q r_{i}\right)+a \sum_{j=0}^{n} \lambda \exp \left(r_{j}-r_{i}\right) \gamma\left(r_{i}-r_{j}\right) y_{j} \\
-a \sum_{j=1}^{n} \gamma\left(r_{j}-r_{i}\right) y_{j}-c \sum_{j=1}^{n} \alpha\left(r_{i}-r_{j}\right) y_{j},
\end{gathered}
$$

where $a=M_{\gamma} d_{0} /\left(k_{0} r_{g}\right), b=d_{0} / r_{g}$, and $c=M_{\alpha} d_{0} /\left(k_{0} r_{g}\right)$. While the nondimensionalized equations were used for our numerical analyses, results below are stated in terms of the more easily interpretable parameters listed in Table 1.

\subsection{Evolutionary dynamics}

The model described above fully determines the population dynamics of a population of $n$ morphs with traits $r_{1}, \ldots, r_{n}$ and population densities $x_{1}, \ldots, x_{n}$. To study evolutionary dynamics, we assume that mutations are sufficiently rare that the population dynamics can be assumed to be close to its demographic attractor when a mutation occurs. From this, we can derive the initial growth rate $S_{r}(m)$ of a rare mutant with trait value $m$ in an environment consisting of a potentially polymorphic resident population with trait values $r=\left(r_{1}, \ldots, r_{n}\right)$. This determination of the mutant fitness is based on the separation of ecological and evolutionary timescales, well known in the genetic literature (e.g. Gillespie, 2004). The function $S_{r}(m)$ of $m$ can be viewed as describing the fitness landscape experienced by the rare mutant. Of special interest are resident trait values $r^{*}$ at which directional selection ceases. At such points, the population can potentially increase its degree of polymorphism, depending on the signs of the curvatures $S_{r^{*}}^{\prime \prime}\left(r_{1}^{*}\right), \ldots, S_{r^{*}}^{\prime \prime}\left(r_{n}^{*}\right)$, of the fitness landscape. We use the magnitude of positive curvatures as a measure of the strength of disruptive selection at such an evolutionary branching point.

To incorporate evolution in the model, we assume that, upon reproduction, the trait value of an individual may differ from that of its parents with a small and constant mutation probability $\tau$. Thus, the rate at which mutations occur in morph $i$ is given by the product of $\tau$ and the reproductive growth rate $B_{i}$ of that 
morph. Trait values of mutants are randomly drawn from a normal distribution around their parent's trait value with standard deviation $\sigma_{m}$. The fate of a mutant depends on its fitness, but is also strongly affected by demographic stochasticity. Based on a well-known result from the theory of birth-death processes, we assume that a mutant successfully invades the population with probability $(b-d) / b$, where $b$ and $d$ are the mutant's birth and death rates (if $b<d$, the invasion probability is 0 ). This corresponds to the probability that a population of individuals that reproduce in discrete time with probability $b$ and die with probability $d$ will persist (see e.g. Grimmett and Stirzaker 1992, pp 272). Since this probability is dimensionless, it remains invariant in the nondimensionalized equations. If a mutant invasion is successful, we determine the resultant coexisting community using a variant of the oligomorphic stochastic model (Ito and Dieckmann, 2007), as described in Appendix

As the evolutionary dynamics in our model result from the invasion of mutants with small random effects, exactly reaching the evolutionary equilibrium, where no further mutant invasion is possible, requires infinite time. We therefore terminate the evolutionary dynamics when the probability that the next mutant would successfully invade is sufficiently low.

\section{Results}

Figure 1a shows how, for a given set of parameters, mutations and selection lead from a single ancestor to the community shown in Fig. 1b, containing eight heterotrophic morphs in addition to the basal autotrophic resource. This result demonstrates that gradual evolution of body size alone suffices to explain the emergence of food webs with high trophic levels. Successive evolutionary branching events allow the community's diversity to grow. These branching events occur at fitness minima, where selection is disruptive and mutants with both higher and lower trait values can thus successfully invade. Although the evolutionary outcome cannot easily be predicted, when trait values and population densities of all resident morphs are known, the strengths of their trophic interactions directly follow from the considered consumption kernel.

Below we first explore how the initial diversification relates to the eventual diversity. Second, we examine what determines the maximum trophic level of the evolved food webs.

\subsection{Conditions promoting initial diversification and even- tual diversity}

Figure 2 shows how the total number of morphs in the evolved food web, and the strength of disruptive selection at the first evolutionary branching point, depend on the following six parameters: $\sigma_{\gamma}$ and $\mu$, which determine the trophic interactions (a, b); $\sigma_{\alpha}$ and $M_{\alpha}$, which determine the competitive interactions $(\mathrm{d}, \mathrm{e})$; and $\lambda$ and $k_{0}$, which determine energy availability $(\mathrm{g}, \mathrm{h})$. The strength of disruptive selection at the first evolutionary branching point is measured as the curvature of the fitness landscape at the point where the population becomes dimorphic.

As Fig. 2 shows, there is a relationship between initial diversification and eventual diversity, with the latter measured by the total number of morphs 
a)

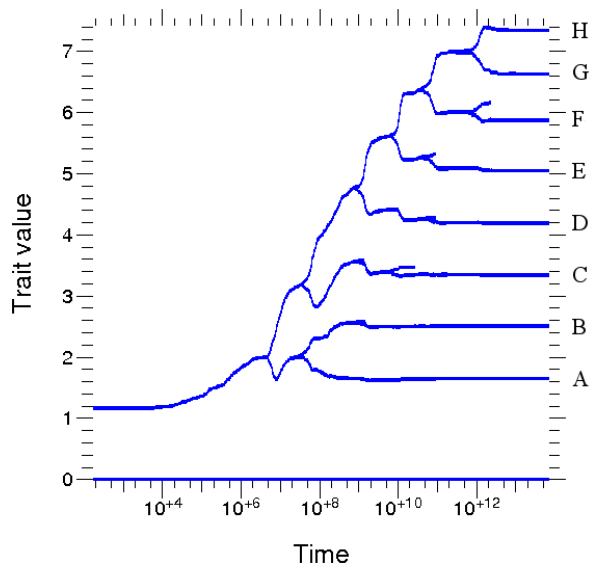

b)

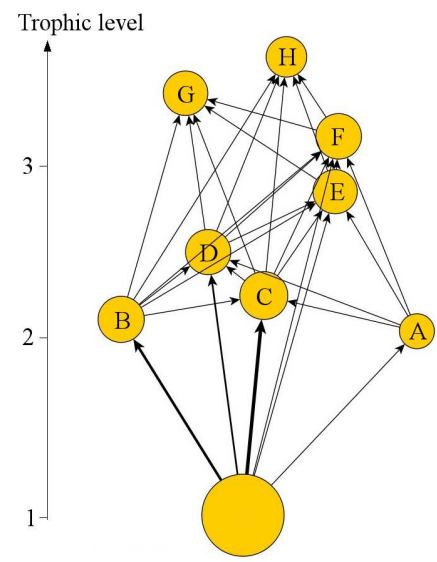

Figure 1: Example of an evolved model food web. a Adaptive radiation and gradual evolution of body sizes, starting from a single ancestor and shown on a logarithmic time scale. The eight heterotrophic morphs in the final community are labeled A-H in order of ascending body size. b Trophic interactions and levels in the final community. Each of the eight heterotrophic morphs A-H is represented by a labeled node and vertically positioned according to its trophic level. The basal autotrophic resource is represented by the large unlabeled node with trophic level 1 . Two nodes are directionally connected from prey to predator if the energy flow between the two corresponding morphs exceeds a threshold value (chosen large enough so that each morph has at least one incoming and one outgoing trophic link). The area of nodes is proportional to the total biomass of the corresponding morph, and the thickness of arrows is proportional to the energy flow between the two corresponding morphs. Parameters: $k_{0}=0.01$, $d_{0}=0.1, \mu=3, M_{\gamma}=10, \sigma_{\gamma}=1.5, \lambda=0.3, q=0.25, M_{K}=1, \sigma_{K}=0.6$, $r_{g}=10, s_{0}=1$, and $\sigma_{m}=0.01$ (implying the non-dimensionalized parameters $a=10, b=0.01$, and $c=1.0)$. 
a)

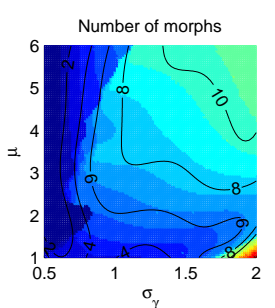

d)

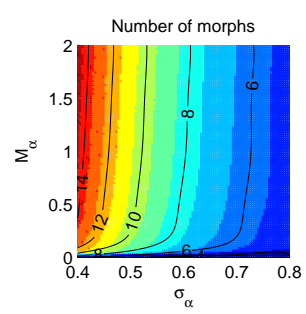

g)

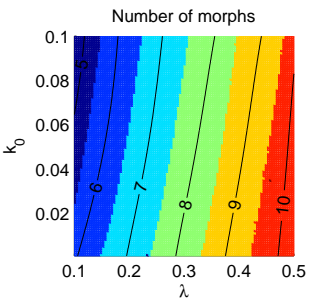

b)

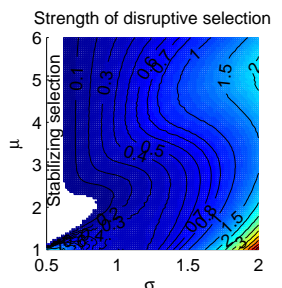

e)

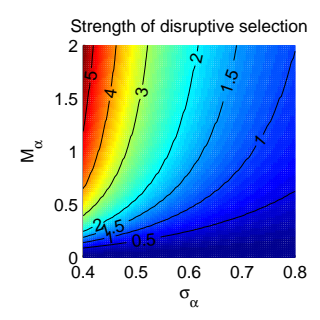

h)

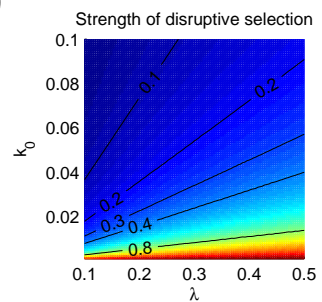

c)

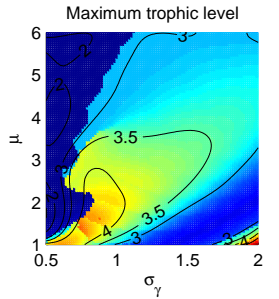

f)

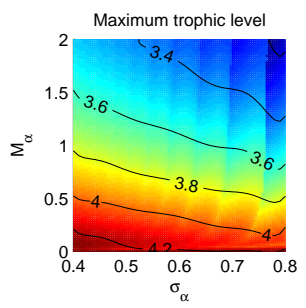

i)

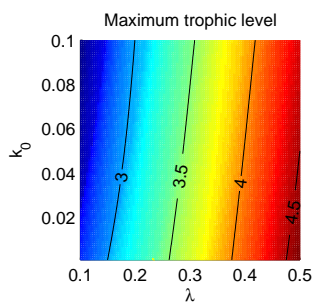

Figure 2: Number of morphs coexisting in the food web at evolutionary equilibrium $(\mathbf{a}, \mathbf{d}, \mathbf{g})$, strength of disruptive selection at the first evolutionary branching point $(\mathbf{b}, \mathbf{e}, \mathbf{h})$, and maximum trophic level $(\mathbf{c}, \mathbf{f}, \mathbf{i})$, for combinations of the mean $\mu$ and standard deviation $\sigma_{\gamma}$ of the consumption kernel $\gamma(\mathbf{a}, \mathbf{b}, \mathbf{c})$, of the amplitude $M_{\alpha}$ and standard deviation $\sigma_{\alpha}$ of the interference-competition kernel $(\mathbf{d}, \mathbf{e}, \mathbf{f})$, and of the conversion efficiency $\lambda$ and the strength $k_{0}$ of density dependence in the resource $(\mathbf{g}, \mathbf{h}, \mathbf{i})$. Colors and contour lines in each panel are based on $100 \times 100$ data points. Contour lines are based on smooth approximations constructed by fitting the data to a high-degree polynomial. The white area in panel $\mathbf{b}$ corresponds to parameter combinations for which selection on the ancestral morph is stabilizing rather than disruptive. Parameters not varied are as in Fig. 1. 
a)

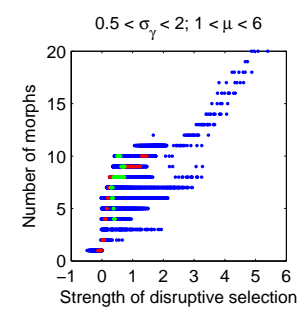

d)

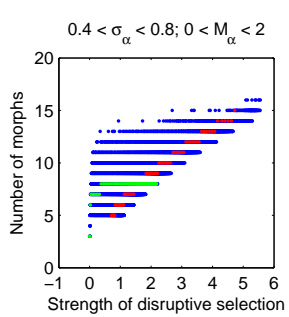

g)

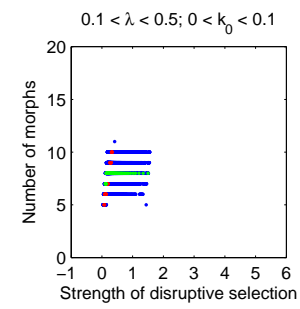

b)

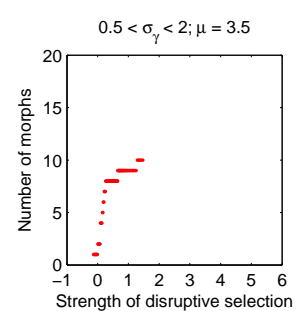

e)

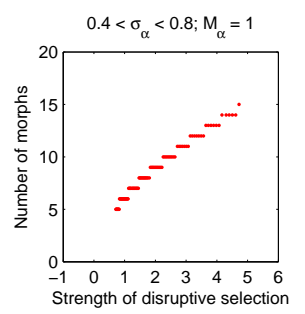

h)

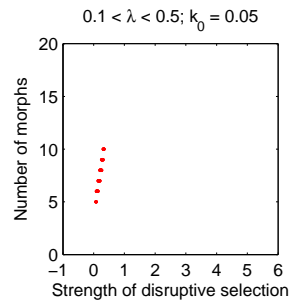

c)

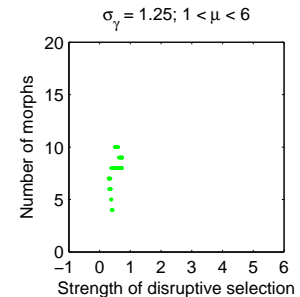

f)

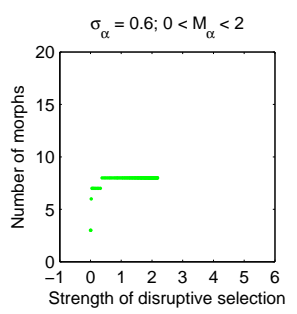

i)

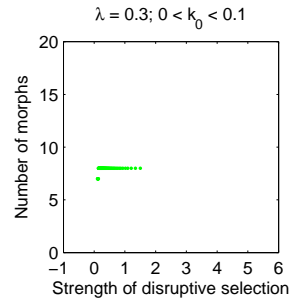

Figure 3: Relationships between the emergence and maintenance of diversity. a, $\mathbf{d}$, $\mathbf{g}$ show combinations of the number of morphs and the strength of disruptive selection for parameter combinations sampled from a uniform grid across the bivariate parameter ranges shown in Fig. 2. The remaining panels show relationships between eventual diversity and initial disruptiveness when only one parameter in these pairs is varied, while the other is being fixed. Note that although the overall relationships are usually positive under bivariate variation (e.g. a, d), variations in only one parameter can reveal positively correlated (e.g. e), uncorrelated (e.g. i), or negatively correlated (e.g. upper and lower parts of $\mathbf{c}$ ) relationships between the emergence and maintenance of diversity. 
evolving in the food web. This relationship is particularly evident for the parameters regulating interference competition: increasing the maximum intensity $M_{\alpha}$ or decreasing the standard deviation $\sigma_{\alpha}$ enlarges the number of evolved morphs and intensifies the strength of disruptive selection (d, e).

For the parameters regulating trophic interactions, the optimal foraging distance $\mu$ and the standard deviation $\sigma_{\gamma}$ of the consumption kernel, a relationship between initial diversification and eventual diversity also exists, but is not as clear. In Fig. 2b, the strength of disruptive selection at the first evolutionary branching point increases towards the bottom-left corner, i.e. towards small values of $\mu$ and $\sigma_{\gamma}$, but there is no corresponding increase in the eventual diversity. The most intense disruptive selection occurs for a low optimal foraging distance $\mu$ and high values of the standard deviation $\sigma_{\gamma}$. This favors the consumption of individuals with larger body size and consequently promotes the build-up of inverted trophic chains, in which smaller individuals derive most of their energy by foraging on individuals with larger body sizes. Such situations are not common in nature, indicating that predation on larger individuals is on average difficult, despite a few existing examples to the contrary.

Figures $2 \mathrm{~g}$ and $2 \mathrm{~h}$ show the relationship between initial diversification and eventual diversity for the conversion efficiency $\lambda$, which determines the loss of energy along each trophic link, and the strength $k_{0}$ of density dependence in the resource, which affects the carrying capacity of the basal autotrophic resource. Both the eventual diversity $(\mathrm{g})$ and the strength of disruptive selection at the first evolutionary branching point (h) increase with the conversion efficiency $\lambda$ and decrease with the strength $k_{0}$ of density dependence in the resource. This is as expected, since a higher conversion efficiency means that more energy is available at high trophic levels, while reduced density dependence in the resource is equivalent to an increased carrying capacity. However, note that the eventual diversity $(\mathrm{g})$ is much more sensitive to variations in $\lambda$ than in $k_{0}$, while the initial diversification (h) is much more sensitive to variations in $k_{0}$ than in $\lambda$.

Figure 3 identifies possible relationships between maintained diversity, measured as the total number of morphs at the evolutionary equilibrium, and emergent diversity, measured as the strength of disruptive selection at the first evolutionary branching point. When parameters are sampled from a uniform grid across the bivariate parameter ranges shown in Fig. 2, the overall relationships between the emergence and the maintenance of diversity are usually positive ( $\mathrm{a}$ and $\mathrm{d}$ ), although this is not always the case (g). The overall positive relationship hides the fact that variations in specific factors may still have opposite consequences for the emergence and maintenance of diversity. Variations in generalism, as measured by the standard deviation $\sigma_{\gamma}$ of the consumption kernel (b), variations in the standard deviation $\sigma_{\alpha}$ of the competition kernel (e), and variations in the conversion efficiency $\lambda(\mathrm{h})$ all create positive relationships between the initial disruptive selection and the eventual number of morphs observed in evolved communities. In contrast, the intensity $M_{\alpha}$ of interference competition (f) and the strength $k_{0}$ of density dependence in the resource (i) have much stronger effects on the emergence of diversity than on its maintenance, thereby creating no obvious link between the two. Finally, note that variations in the optimal foraging distance $\mu$ strongly affect the maintenance of diversity, without much affecting the initial disruptive selection (c). Such variations may even yield a negative relationship between the emergence and maintenance of diversity if only part of the variation is considered. In summary, 
Fig. 3 clearly shows that a parameter that favors the emergence of diversity does not necessarily promote the maintenance of diversity in evolved communities, and vice versa.

\subsection{Conditions promoting high trophic levels}

Figure 2c shows how the maximum trophic level in the evolved food webs depends on the foraging parameters $\mu$ and $\sigma_{\gamma}$. The highest trophic levels are obtained in food webs with low to medium diversity. However, high trophic levels are also obtained in food webs with high diversity. A comparison with the eventual diversity in Fig. 2a shows that diverse food webs with 3-4 trophic levels are generally obtained when the ratio $\mu / \sigma_{\gamma}$ ranges between 1 and 4 . For smaller ratios, cannibalism becomes important in restricting population densities. And for very small ratios, runaway selection towards smaller body sizes occurs. On the other hand, when the ratio is very large, the food web cannot build up through gradual evolution and evolutionary branching, as the evolving morphs would have to pass through body sizes for which no prey is located within the range of body sizes that can efficiently be consumed.

Figure 2f shows how the maximum trophic level depends on the parameters determining competitive interactions. Less intense interference competition (small $M_{\alpha}$ ) and interference competition that mainly affects individuals of similar size (small $\sigma_{\alpha}$ ) result in evolved food webs with higher maximum trophic levels. This can be contrasted with the increase of eventual diversity with competitive intensity $M_{\alpha}$ shown in Fig. 2d. At first sight, this contrast may seem counterintuitive; however, intense interference competition among the heterotrophic morphs leads to their having very small population densities relative to the basal autotrophic resource. Hence, under intense interference competition, even morphs with large body sizes can potentially derive a large share of their energy intake directly from the resource, thus reducing the maximum trophic level.

Finally, as shown in Fig. 2i, the maximum trophic level of the evolved food webs increases with the conversion efficiency and decreases with the strength of density dependence in the resource. This is the same pattern seen in Fig. $2 \mathrm{~g}$ for the eventual diversity, which indicates that on an evolutionary timescale increased energy availability has a clear and positive effect on the evolved biodiversity and on food-chain lengths.

\section{Discussion}

Several experimental and empirical studies have recently explored conditions for initial diversification in bacteria and insects. These studies have identified relevant factors such as spatial structure (Rainey and Travisano 1998; Habets 2006; Stocks and McPeek 2006), parasitism (Buckling and Rainey 2002b), competition (Friesen et al., 2004; McLean et al., 2005), predation (Nosil and Crespi, 2006), adaptation to dry conditions (Klak, 2004), energetic input (Kassen and Rainey, 2004), and ecological disturbance (Kassen and Rainey, 2004; Massin and Gonzalez, 2006). The increasing understanding of factors and conditions that favor initial diversification begets the question whether these same factors and conditions also promote the emergence of diverse ecological communities. If this could consistently be shown, insights from laboratory studies on initial 
diversification would gain immediate relevance for the management of biodiversity.

By comparing the strength of disruptive selection at the first evolutionary branching point with the number of morphs in the finally evolved community for the food-web model considered here, we could establish that even though the effects of conditions on initial diversification and eventual diversity are often positively correlated, no clear relationship between the two may exist when focusing on variations in individual factors. To our knowledge, our study is the first explicitly to address the relationship between disruptive selection and the maintenance of diversity within communities. We have identified factors that affect the emergence and maintenance of diversity in a similar fashion, but we have also shown that such positive relationships are by no means necessary, as some factors may largely constrain the emergence of diversity while being almost neutral with respect to its maintenance, or vice versa.

We further studied how interaction parameters and energy availability affected the maximum trophic level of the evolved community. Although explicit theoretical investigations elucidating the link between total diversity and food-chain length are lacking, constraints affecting either of these community characteristics are well known and may be used to infer a link. For instance, food-chain length is generally thought to increase with productivity (Oksanen et al., 1981; Persson et al., 1992; Abrams, 1993; Jennings and Warr, 2003). The relationship between diversity and productivity is thought to be positive at regional scales and unimodal at local scales (Steiner and Leibold, 2004; Chase and Leibold, 2002). One would therefore expect that diversity and food-chain length are positively related at regional scales or at local scales in poor environments, while the correlation between the two could be negative at local scales in rich environments. Another important covariate is ecosystem size. Ecosystem size is known to be positively correlated with food-chain length (Post et al., 2000; Jennings and Warr, 2003; Brose et al., 2004) and the average number of species (Macarthur and Wilson, 2001; Rosenzweig, 1995; Brose et al., 2004). Because of this covariation, food-chain length is predicted to increase with species diversity.

Rather unexpectedly, therefore, we found that the most diverse food webs we obtained based on our model did not have the highest trophic levels, even though the diverse food webs under study generally had 3-4 trophic levels. Interestingly, we found that while small amounts of interference competition resulted in food webs with high trophic levels, an intermediate maximum is quickly reached beyond which a further increase in interference competition tends to reduce the number of trophic levels (whereas the number of evolved morphs may still continue to increase). This effect was also noticed and discussed by Loeuille and Loreau (2005). The food-web model introduced in this study adds to a rapidly growing family of models of community evolution that aim at understanding trophic structure through assembly rules mimicking mutation (Caldarelli et al. 1998; Drossel et al. 2001; McKane 2004; Tokita and Yasutomi 2006; Ito and Ikegami 2006; Rossberg et al. 2005, 2006, 2008; Loeuille and Loreau 2005; Christensen et al. 2002; Yoshida 2003; Bastolla et al. 2005 reviewed in Loeuille and Loreau 2009). Although all of these models could potentially be used to assess the relationship between the emergence and maintenance of diversity, the lack of gradual evolution in most models makes it difficult to quantify conditions for initial diversification. As far as we know, we are the first explicitly to make this comparison. 
The study most similar to ours is Loeuille and Loreau (2005), as our model and study are a natural extension of this earlier work. Major changes are that interactions are now expressed in terms of relative instead of absolute differences in body size, and that interference competition is now modeled through a Gaussian function. These models support some common results. For example, both in Loeuille and Loreau (2005) and in the present study, food-chain length and eventual diversity were very sensitive to nutrient supply and conversion efficiency. Finding such concordant results in two different studies highlights their robustness with respect to the changes we made in the description of trophic and competitive interactions.

The work presented here can be extended in several ways. For example, the effect on food-web structure of introducing several autotrophic morphs could be investigated, and more complicated assumptions about density-dependent or trait-dependent encounter rates could be explored. Another interesting extension would be to incorporate a higher-dimensional trait space in which predation preference could evolve, while still retaining the allometric scaling relationships that characterize the effects of body size. A particularly promising extension would be to use the model presented here to study the ecological and evolutionary impacts of anthropogenic change on biodiversity. For example, understanding the impacts of size-selective harvesting is paramount for assessing the potential detrimental implications of modern industrial fisheries. Communityevolution models such as the one presented here allow considering community effects, evolutionary effects, and their interactions, all of which are highly relevant for understanding how exploitation affects ecosystems.

\section{Acknowledgements}

We thank Hiroshi C. Ito, Jacob Johansson, Shovonlal Roy, and Jack Teng for valuable comments and suggestions. This research has been supported by the European Marie Curie Research Training Network FishACE (Fisheries-induced Adaptive Changes in Exploited Stocks), funded through the European Community's Sixth Framework Programme (Contract MRTN-CT-2004-005578). In addition, A. B. gratefully acknowledges support by the Swedish Kempe Foundations and the Japan Society for the Promotion of Science. U. D. gratefully acknowledges support by the European Science Foundation, the Austrian Science Fund, and the Vienna Science and Technology Fund. Michel Loreau gratefully acknowledges support by the Natural Sciences and Engineering Research Council of Canada and the Canada Research Chairs program.

\section{A Appendix}

\subsection{A.1 Evolutionary dynamics}

To increase computational efficiency, we have implemented the evolutionary process such that for any community of coexisting morphs we draw the next successfully invading mutant trait value and the time at which the invasion occurs. 


\subsubsection{A.1.1 Determining the rate of successful mutations}

We first determine the probability $p_{i}$ that a mutant in morph $i$ is successful. Recalling that we assume a mutant's trait value to be normally distributed with variance $\sigma_{m}^{2}$ around its parent's trait value, and denoting by $p_{r}(m)$ the probability that a mutant with trait value $m$ successfully invades a community characterized by the trait values $r=\left(r_{1}, \ldots, r_{n}\right)$ of the resident morph, $p_{i}$ is given by

$$
p_{i}=\frac{1}{\sigma_{m}} \int_{-\infty}^{\infty} \phi\left(\frac{m-r_{i}}{\sigma_{m}}\right) p_{r}(m) d m
$$

where $\phi$ is the probability density function of the standard normal distribution. In general, this integral would have to be evaluated numerically, but for small values of $\sigma_{m}$, a second-order Taylor approximation of $p_{r}(m)$ around $r_{i}$ can be used. With $\tau B_{i}$ denoting the rate at which mutations occur in morph $i$, the rate at which successful mutations occur in morph $i$ is $s_{i}=\tau B_{i} p_{i}$.

\subsubsection{A.1.2 Drawing the next successful mutant}

The morph $i$ in which the next successful mutant occurs is drawn with probability $s_{i} / S$, with $S=\sum_{i} s_{i}$. We then draw the mutant's trait value $m$ from the probability density function

$$
f(m)=\frac{1}{\sigma_{m} p_{i}} \phi\left(\frac{m-r_{i}}{\sigma_{m}}\right) p_{r}(m) .
$$

To do this, we use the rejection method described in Press et al. (1992). After choosing a sufficiently large maximal value $D$ of the deviation $\left|m-r_{i}\right|$, and after determining an upper bound $F(m)$ so that $F(m)>f(m)$ for all $m$, we draw a uniformly distributed bivariate random deviate $(m, y)$ with $\left|m-r_{i}\right|<D$ and $y<F(m)$. If $y<f(m)$, we take $m$ as the new mutant trait value; otherwise, the bivariate random draw is repeated. Alternatively, we can draw $m$ from the normal distribution $\varphi\left(\left(m-r_{i}\right) / \sigma_{m}\right) / \sigma_{m}$ and $y$ from the uniform distribution over the unit interval. If $y<p_{r}(m)$, we take $m$ as the new mutant trait value; otherwise, the bivariate random draw is repeated. Finally, since the arrival of successful mutants follows a Poisson process with intensity $S$, the waiting time between such events is drawn from an exponential distribution with parameter $S$ (see e.g. Grimmett and Stirzaker, 1992, pp 248).

\subsection{A.1.3 Implementing the oligomorphic stochastic model}

The evolutionary dynamics of the oligomorphic stochastic model (Ito and Dieckmann, 2007) are obtained by assuming a timescale separation between population dynamics and evolutionary dynamics and by making a simplifying assumption for the conditions under which a mutant trait value replaces the resident trait value from which it arises. Specifically, we implemented a variant of the oligomorphic stochastic model that considers a community viable if the corresponding system of Lotka-Volterra equations has an interior rest point (see Hofbauer and Sigmund (1998) for a thorough account of the relationship between Lotka-Volterra dynamics and the existence of interior rest points).

It is worth noting that the method described by Law and Morton (1996) for species-assembly models based on Lotka-Volterra dynamics - in particular, 
the use of permanence to determine a community's viability - can possibly be adapted to evolutionary models based on Lotka-Volterra dynamics, which would enable an improvement over the algorithm described here.

\subsection{A.2 Definition of trophic levels}

Although the concept of trophic level is frequently encountered in the literature, it is not always easy to define. Most of the proposed methods (see Yodzis, 1989, for examples) are either difficult to apply to food webs containing cycles and loops, or expensive to calculate in practice. Furthermore, if trophic levels are defined as taking integer values only, the implied abrupt jumps in trophic level will make it difficult to track the dynamics of evolving food webs. Following Odum and Heald (1975) and Adams et al. (1983), we define a morph's realvalued fractional trophic level as 1 plus the average trophic level of its prey. Denoting by $t_{i}$ the fractional trophic level of morph $i=0, \ldots, n$ (with $t_{0}=1$ corresponding to the basal autotrophic resource) and by $v_{i j}$ the proportion of energy entering morph $i$ from morph $j$ (with $\sum_{j} v_{i j}=1$ ), the fractional trophic levels $t_{i}$ are defined as the solutions of the linear equations

$$
t_{i}=1+\sum_{j=0}^{n} v_{i j} t_{j} .
$$

\section{References}

Abrams PA (1993) Effect of increased productivity on the abundances of trophic levels. Am Nat 141:351-371

Abrams PA (1997) Evolutionary responses of foraging-related traits in unstable predator-prey systems. Evol Ecol 11:673-686

Adams SM, Kimmel BL, Ploskey GR (1983) Sources of organic matter for reservoir fish production: a trophic-dynamics analysis. Can J Fish Aquat Sci 40:1480-1495

Bastolla U, Lässig M, Manrubia SC, Valleriani A (2005) Biodiversity in model ecosystems, ii: species assembly and food web structure. J Theor Biol 235:531-539

Bergelson J, Dwyer G, Emerson J (2001) Models and data on plant-enemy coevolution. Annu Rev Genet 35:469-499

Brose U, Osling A, Harrison K, Martinez ND (2004) Unified spatial scaling of species and their trophic interactions. Nature 428:167-171

Buckling A, Rainey PB (2002a) Antagonistic coevolution between a bacterium and a bacteriophage. Proc R Soc Lond B 269:931-936

Buckling A, Rainey PB (2002b) The role of parasites in sympatric and allopatric host diversification. Nature 420:496-499

Caldarelli G, Higgs P, McKane A (1998) Modelling coevolution in multispecies communities. J Theor Biol 193:345-358 
Cattin M, Bersier L, Banasek-Richter C, Baltensperger R, Gabriel J (2004) Phylogenetic constraints and adaptation explain food web structure. Nature 427:835-839

Chase JM, Leibold MA (2002) Spatial scale dictates the productivitybiodiversity relationship. Nature 416:427-430

Christensen K, di Collobiano SA, Hall M, Jensen HJ (2002) Tangled nature: a model of evolutionary ecology. J Theor Biol 216:73-84

Cohen J, Briand F, Newman C (1990) Community food webs: data and theory. Springer Verlag, Berlin

Díaz S, Cabido M (2001) Vive la différence: plant functional diversity matters to ecosystem processes. Trends Ecol Evol 16:646-655

Dieckmann U, Law R (1996) The dynamical theory of coevolution: a derivation from stochastic ecological processes. Trends Ecol Evol 34:579-612

Drake JA (1990) The mechanics of community assembly and succession. J Theor Biol 147:213-233

Drossel B, Higgs P, McKane A (2001) The influence of predator-prey population dynamics on the long-term evolution of food web structure. J Theor Biol 208:91-107

Friesen M, Saxer G, Travisano M, Doebeli M (2004) Experimental evidence for sympatric ecological diversification due to frequency-dependent competition in Escherichia coli. Evolution 58(2):245-260

Geritz SAH, Kisdi E, Meszéna G, Metz JAJ (1998) Evolutionary singular strategies and the adaptive growth and branching of the evolutionary tree. Evol Ecol $12: 35-57$

Gillespie JH (2004) Population genetics: a concise guide. The John Hopkins University Press

Grimmett GR, Stirzaker DR (1992) Probability and random processes, 2nd edn. The Clarendon Press Oxford University Press, New York

Habets MGJL, Rozen DE, Hoekstra RF, Arjan J, De Visser AGM (2006) The effect of population structure on the adaptive radiation of microbial populations evolving in spatially structured environments. Ecol Lett 9:1041-1048

Hector A, Schmid B, Beierkuhnlein C, Caldeira MC, Diemer M, Dimitrakopoulos PG, Finn JA, Freitas H, Giller PS, Good J, Harris R, Högberg P, HussDanell K, Joshi J, Jumpponen A, Körner C, Leadley PW, Loreau M, Minns A, Mulder CPH, O'Donovan G, Otway SJ, Pereira JS, Prinz A, Read DJ, Scherer-Lorenzen M, Schulze ED, Siamantziouras ASD, Spehn EM, Terry AC, Troumbis AY, Woodward FI, Yachi S, Lawton JH (1999) Plant diversity and productivity experiments in european grasslands. Science 286:1123-1127

Hofbauer J, Sigmund K (1998) Evolutionary games and population dynamics. Cambridge University Press, Cambridge, UK 
Hooper DU, Chapin FS, Ewel JJ, Hector A, Inchausti P, Lavorel S, Lawton JH, Lodge DM, Loreau M, Naeem S, Schmid B, Setl H, Symstad AJ, Vandermeer J, Wardle DA (2005) Effects of biodiversity on ecosystem functioning: a consensus of current knowledge. Ecol Monogr 75:3-35

Ito HC, Dieckmann U (2007) A new mechanism for recurrent adaptive radiations. Am Nat 170:E96-E111

Ito HC, Ikegami T (2006) Food-web formation with recursive evolutionary branching. J Theor Biol 238:1-10

Ives AR, Klug JL, Gross K (2000) Stability and species richness in complex communities. Ecol Lett 3:399-411

Jansen VAA, Kokkoris GD (2003) Complexity and stability revisited. Ecol Lett 6:498-502

Jennings S, Warr KJ (2003) Smaller predator-prey size ratios in longer food chains. Proc R Soc Lond B 270:1413-1417

Kassen R, Rainey PB (2004) The ecology and genetics of microbial diversity. Annu Rev Microbiol 58:207-231

Kinzig AP, Pacala SW, Tilman D (eds) (2001) The functional consequences of biodiversity: empirical progress and theoretical extensions. Princeton University Press

Klak C (2004) Unmatched tempo of evolution in southern african semi-desert ice plants. Nature 427:63-65

Kondoh M (2003) Foraging adaptation and the relationship between food-web complexity and stability. Science 299:1388-1391

Law R, Morton D (1996) Permanence and the assembly of ecological communities. Ecology 77(3):762-775

Loeuille N, Loreau M (2009) Emergence of complex food web structure in community evolution models. In: Verhoef HA, Morin PJ (eds) Community ecology, Oxford University Press

Loeuille N, Loreau M (2005) Evolutionary emergence of size structured food webs. Proc Natl Acad Sci USA 102(16):5761-5766

Loeuille N, Loreau M, Ferrière R (2002) Consequences of plant-herbivore coevolution on the dynamics and functioning of ecosystems. J Theor Biol 217:369 381

Loreau M, Naeem S, Inchausti P, Bengtsson J, Grime JP, Hector A, Hooper DU, Huston MA, Raffaelli D, Schmid B, Tilman D, Wardle DA (2001) Biodiversity and ecosystem functioning: current knowledge and future challenges. Science 294:804-808

Loreau M, Naeem S, Inchausti P (eds)(2002) Biodiversity and ecosystem functioning: synthesis and perspectives. Oxford University Press 
Macarthur RH, Wilson EO (2001) The theory of island biogeography. Princeton University Press

Massin N, Gonzalez A (2006) Adaptive radiation in a fluctuating environment: disturbance affects the evolution of diversity in a bacterial microcosm. Evol Ecol Res 8:471-481

May RM (1972) Will a large complex system be stable? Nature 238:413-414

McKane AJ (2004) Evolving complex food webs. Eur Phys J B 38:287-295

McLean RC, Dickson A, Bell G (2005) Resource competition and adaptive radiation in a microbial microcosm. Ecol Lett 8:38-46

Morton D, Law R (1997) Regional species pools and the assembly of local ecological communities. J Theor Biol 187:321-331

Naeem S, Thompson LJ, Lawler SP, Lawton JH, Woodfin RM (1994) Declining biodiversity can alter the performance of ecosystems. Nature 368:734-737

Naeem S, Bunker DE, Hector A, Loreau M, Perrings C (eds) (2009) Biodiversity, ecosystem functioning, and human wellbeing: an ecological and economic perspective. Oxford University Press

Nosil P, Crespi BJ (2006) Experimental evidence that predation promotes divergence in adaptive radiation. Proc Natl Acad Sci USA 103:9090-9095

Odum WE, Heald EJ (1975) The detritus-based food web of an estuarine mangrove community. In: Cronin L (ed) Estuarine research. Chemistry biology and the estuarine system, vol 1, Academic Press, London

Oksanen L, Fretwell SD, Arruda J, Niemela P (1981) Exploitation ecosystems in gradients of primary productivity. Am Nat 118:240-261

Persson L, Diehl S, Johansson L, Hamrin SF (1992) Interactions in temperate lake ecosystems: a test of food chain theory. Am Nat 140:59-84

Peters RH (1983) The ecological implications of body size. Press Syndicate of the University Of Cambridge, Cambridge, UK

Pimm SL, Lawton JH (1977) The number of trophic levels in ecological communities. Nature 268:329-331

Post DM, Pace ML, Hairston NGJ (2000) Ecosystem size determines food chain length in lakes. Nature 405:1047-1049

Post WM, Pimm SL (1983) Community assembly and food web stability. Math Biosci 64:169-192

Press WH, Teukolsky SA, Vetterling WT, William Y, Flannery BP (1992) Numerical recipes in C, 2nd edn. Cambridge University Press, Cambridge, the art of scientific computing

Rainey PB, Travisano M (1998) Adaptive radiation in a heterogeneous environment. Nature 394:6973 
Rosenzweig ML (1995) Species diversity in space and time. Cambridge University Press

Rossberg AG, Matsuda H, Amemiya T, Itoh K (2005) An explanatory model for foodweb structure and evolution. Ecological Complexity 312:312-321

Rossberg AG, Matsuda H, Amemiya T, Itoh K (2006) Food webs: experts consuming families of experts. J Theor Biol 241:552-563

Rossberg AG, Ishii R, Amemiya T, Itoh K (2008) The top-down mechanism for body mass-abundance scaling. Ecology 89(2):567-580

Saloniemi I (1993) A coevolutionary predator-prey model with quantitative characters. Am Nat 141(6):880-896

Steiner CF, Leibold MA (2004) Cyclic assembly trajectories and scale-dependent productivity-diversity relationship. Ecology 85:107-113

Stocks R, McPeek MA (2006) A tale of two diversitfications: reciprocal habitat shifts to fill ecological space along the pond permanence gradient. Am Nat 168:50-72

Tilman D, Wedin D, J K (1996) Productivity and sustainability influenced by biodiversity in grassland ecosystems. Nature 379:718-720

Tokita K, Yasutomi A (2006) Emergence of a complex and stable network in a model ecosystem with extinction and mutation. Theor Popul Biol 63:131-146

Williams RJ, Martinez ND (2000) Simple rules yield complex food webs. Nature 404:180-183

Worm B, Duffy JE (2003) Biodiversity, productivity and stability in real food webs. Trends Ecol Evol 18(12):628-632

Yodzis P (1989) Introduction to theoretical ecology. Harper \& Row

Yoshida K (2003) Dynamics of evolutionary patterns of clades in a food web system model. Ecol Res 18:625-637 